\title{
Fabrication and modification of implantable optrode arrays for in vivo optogenetic applications
}

\author{
Lulu Wang ${ }^{1,2}$, Kang Huang ${ }^{1,2}$, Cheng Zhong ${ }^{1}$, Liping Wang ${ }^{1 凶}$, Yi Lu ${ }^{1 \bowtie}$ \\ ${ }^{1}$ Shenzhen Key Lab of Neuropsychiatric Modulation and Collaborative Innovation Center for Brain Science, CAS \\ Center for Excellence in Brain Science and Intelligence Technology, The Brain Cognition and Brain Disease \\ Institute, Shenzhen Institutes of Advanced Technology, Chinese Academy of Sciences, Shenzhen 518055, China \\ 2 Shenzhen College of Advanced Technology, University of Chinese Academy of Sciences, Shenzhen 518055, China
}

Received: 3 March 2018 / Accepted: 20 March 2018 / Published online: 20 April 2018

\section{Graphical Abstract}
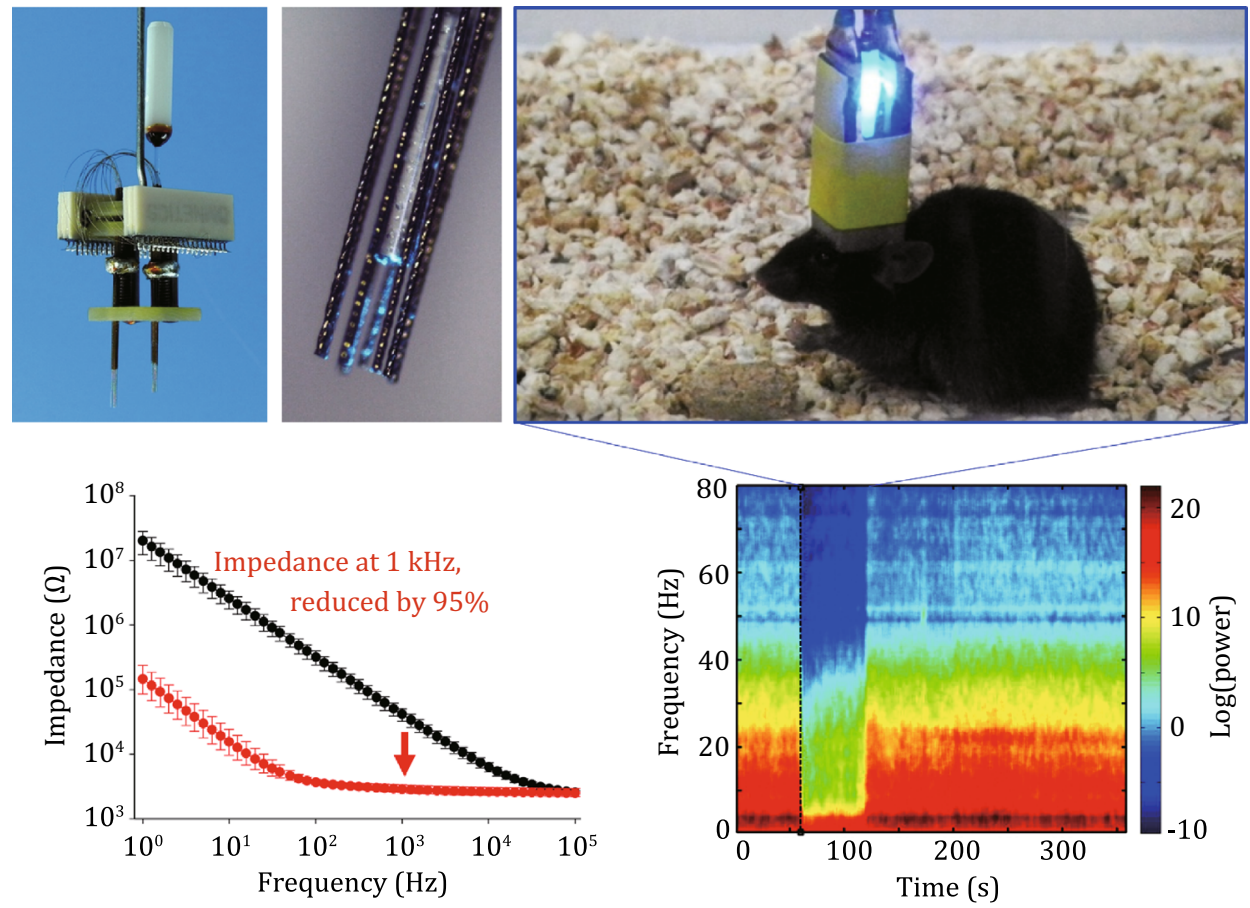

Abstract Recent advances in optogenetics have established a precisely timed and cell-specific methodology for understanding the functions of brain circuits and the mechanisms underlying neuropsychiatric disorders. However, the fabrication of optrodes, a key functional element in optogenetics, remains a great challenge. Here, we report reliable and efficient fabrication strategies for chronically

Lulu Wang and Kang Huang have contributed equally to this work.

$\bowtie$ Correspondence: lp.wang@siat.ac.cn (L. Wang),

luyi@siat.ac.cn (Y. Lu) 
implantable optrode arrays. To improve the performance of the fabricated optrode arrays, surfaces of the recording sites were modified using optimized electrochemical processes. We have also demonstrated the feasibility of using the fabricated optrode arrays to detect seizures in multiple brain regions and inhibit ictal propagation in vivo. Furthermore, the results of the histology study imply that the electrodeposition of composite conducting polymers notably alleviated the inflammatory response and improved neuronal survival at the implant/neural-tissue interface. In summary, we provide reliable and efficient strategies for the fabrication and modification of customized optrode arrays that can fulfill the requirements of in vivo optogenetic applications.

Keywords Optogenetics, Optrode, Neural electrode, Electrodeposition, Optical stimulation, Electrophysiological recording

\section{INTRODUCTION}

Optogenetics is a technology that combines optical control and genetic targeting using cell-type-specific and optically sensitive proteins for the precise manipulation of neuronal functions with millisecond precision (Yizhar et al. 2011a; Zhang et al. 2007). A major advantage of optogenetic methodology is that it enables the excitation or inhibition of specific neuron subtypes and the electrophysiological recording of neuronal activity simultaneously without causing large stimulus artifacts that may overlap the recording results (Anikeeva et al. 2012). Benefiting from these advantages, optogenetics has been widely applied to investigate the mechanisms of neuropsychiatric diseases as well as the functions of brain networks through precisely timed control of specific neuron groups in a neural circuit (Brown 2012; Gradinaru 2009; Kravitz et al. 2010; Li 2015; Lu et al. 2016; Otis et al. 2017; Schmitt et al. 2017; Tovote et al. 2016; Tye et al. 2013; Yizhar et al. 2011b; Zimmerman et al. 2016).

In order to investigate complex brain processing mechanisms at a functional level, researchers need to use optogenetics to detect the activity from specific neural circuits during the manipulation of target neuron populations. In many cases, chronic studies in freely moving animals are necessary for dissecting the characteristics of a particular neural circuit control animal behavior and understanding the intrinsic neural basis of a specific behavioral phenomenon. Therefore, chronically implantable optrode arrays that integrate optical stimulation with large-scale electrophysiological readout methods are greatly desired for in vivo applications (Anikeeva et al. 2012; Lu et al. 2012). Recent advances in Micro-Electro-Mechanical Systems (MEMS) have shed new light on the development of optrodes (Chen et al. 2013; Dehkhoda et al. 2018; Iseri and Kuzum 2017; Kwon et al. 2015). However, a reliable and efficient fabrication technology for customized chronically implantable optrodes still needs to be developed to meet the requirements of different experimental purposes.

Optrode arrays are typically composed of an optical waveguide for light delivery and multiple electrode channels for recording. The electrode is recognized to be a crucial readout element in optrodes, which determines the signal-recording quality required for neural circuit dissection (Anikeeva et al. 2012; Lu et al. 2012). However, achieving effective and stable long-term electrophysiological recording, with the electrode in vivo, continues to be a great challenge. Therefore, another desirable research goal related to optrodes is to develop novel materials for biocompatible and lowimpedance electrode/neural-tissue interfaces.

With the development of microfabrication technologies, high-density microelectrode arrays have been widely used to increase spatial precision and minimize insertion trauma. However, the impedance of the electrode increases drastically with a decrease in its size, which subsequently results in high thermal-background noise and a low signal-to-noise ratio during recording. To solve this problem, electrochemical deposition of high double-layer capacitance or Faradaic-capacitance materials, such as platinum particles, titanium nitride, and iridium oxide, has been frequently used to reduce the electrode impedance (Cogan 2008; Kotov et al. 2009; Lu et al. 2009b). Another major bottleneck that unfortunately hinders the applications of neural probes is the inconsistent performance caused by elicited inflammatory response (Grill et al. 2009; Kotov et al. 2009; Schwartz et al. 2006). The inflammatory response usually results in a dense astroglial encapsulation of the implant, which isolates the neural probe from the surrounding neurons, and the response also leads to a loss of neurons adjacent to the electrode/neural-tissue interface, which further deteriorates the performance of the electrode in chronic studies. A commonly applied strategy for improving both the electrochemical performance and biocompatibility of neural electrodes is surface 
modification with conducting polymers (CPs). Previous studies have reported that CPs such as polypyrrole (PPy) and poly(3,4-ethylenedioxythiophene) (PEDOT) can notably increase the electrode capacitance, reduce surface impedance, and alleviate inflammatory responses (Abidian and Martin 2008; George et al. 2005; Lu et al. 2010; Zhong et al. 2017). However, the electrodeposition process and characteristics of these materials have not been adequately investigated with respect to the requirements of optogenetics.

In this work, we report on the fabrication of two types of optrode arrays for in vivo optogenetic applications, including a microwire optrode array for investigating single brain regions and a drivable optrode array for simultaneously targeting multiple brain structures. In order to improve the performance of the fabricated optrodes during electrophysiological recording, we also demonstrate three different strategies for decreasing the electrode impedance: a simplified cathodic electrochemical process for the deposition of platinum particles, a cyclic voltammetric process for depositing iridium oxide, and a multi-step modification process for composite CP deposition. The electrochemical characteristics and surface morphology of the deposited optrodes were determined by performing cyclic voltammetry (CV), electrochemical impedance spectroscopy (EIS), and scanning electron microscopy (SEM) analyses. Furthermore, we examined the feasibility of using customized optrode arrays to investigate the neuronal activity during the ictal period and inhibit seizure propagation in multiple brain regions in vivo. Finally, after chronic implantation in rat brains, the astrocyte intensity and neuronal survival around the modified implant sites were assessed by analyzing the immunoreactivity of glial fibrillary acidic protein (GFAP) and neuronal nuclei (NeuN), respectively. All of these results were evaluated and discussed with respect to the requirements of optogenetic applications.

\section{EXPERIMENTAL SECTION}

\section{Animals}

All experiments were conducted in accordance with the protocols approved by the Ethics Committee for Animal Research, Shenzhen Institute of Advanced Technology, Chinese Academy of Sciences. Male Sprague-Dawley (SD) rats weighting 240-280 g were used for optrode implantation and histological study. Adult male VGATChR2(H134R)-EYFP bacterial artificial chromosome (BAC) transgenic mice were used for optogenetic stimulation and electrophysiological recordings. Animals were housed in controlled conditions (ambient temperature $24 \pm 1{ }^{\circ} \mathrm{C}$, humidity $50 \%-60 \%$, 12 -h light/dark cycle) with food and water given ad libitum.

\section{Fabrication of optogenetic probes}

\section{Fabrication of microwire optrode arrays}

Microwire optrode arrays were fabricated from optical fibers (diameter $200 \mu \mathrm{m}$, NA 0.37, Thorlabs, USA) and formvar-coated nickel-chromium (diameter $35.6 \mu \mathrm{m}$, Stablohm 650, California Fine Wire, USA) or platinumiridium (diameter $35.6 \mu \mathrm{m}, \mathrm{Pt} / \mathrm{Ir}$ 90/10, California Fine Wire, USA) microwires using a custom-made optrode mold (Fig. 1A). Eight microwires in an optrode array were arranged in two parallel rows for electrophysiological recordings, with each row containing four wires. The spacing between neighboring microwires was $180 \mu \mathrm{m}$. Approximately $2 \mathrm{~mm}$ of the formvar coating was removed from one end of each microwire by brief exposure to a flame, and each microwire was soldered into separate slots of a standard electrode connector (Omnetics, USA). Two pairs of uninsulated silver microwires (diameter $100 \mu \mathrm{m}$ ) were soldered into the electrode connector as the reference and ground electrodes, respectively. An optical fiber was arranged to deliver light using the optrode mold. One end of the optical fiber was fixed to an optical ceramic ferrule (inner diameter $=220 \mu \mathrm{m}$ ) and stabilized onto the electrode connector using epoxy resin (Fig. 1B). The tip of the optrode was coated with polyethylene glycol (PEG, $\quad M_{\mathrm{W}}=2000 \mathrm{~g} / \mathrm{mol}$, Sigma-Aldrich, USA) to enhance its mechanical strength (Fig. 1C). Prior to implantation, phosphate-buffered saline (PBS) was applied to dissolve and remove the PEG coatings. In order to ensure illumination of the neurons to be recorded, the tips of the microwires were placed approximately $500 \mu \mathrm{m}$ deeper than the optical fiber (Fig. 1D). This microwire optrode array is easy to fabricate and is suitable for targeting single regions in relatively shallow brain structures (Fig. 1E).

\section{Fabrication of multisite drivable optrode arrays}

The drivable optrode arrays consisted of screw-based microdrive scaffolds (Fig. 2A) and optrode bundles (Fig. 2B). Each optrode bundle, which contains one stimulation channel and eight twisted tetrodes (32 electrophysiological recording channels in total), was fabricated from optical fibers (diameter $200 \mu \mathrm{m}$, NA 0.37, Thorlabs, USA) and formvar-coated nickelchromium (diameter 12.7 or $17.8 \mu \mathrm{m}$, Stablohm 650, California Fine Wire, USA) or platinum-iridium (diameter 12.7 or $17.8 \mu \mathrm{m}, \mathrm{Pt} / \mathrm{Ir}$ 80/20, California Fine Wire, USA) 

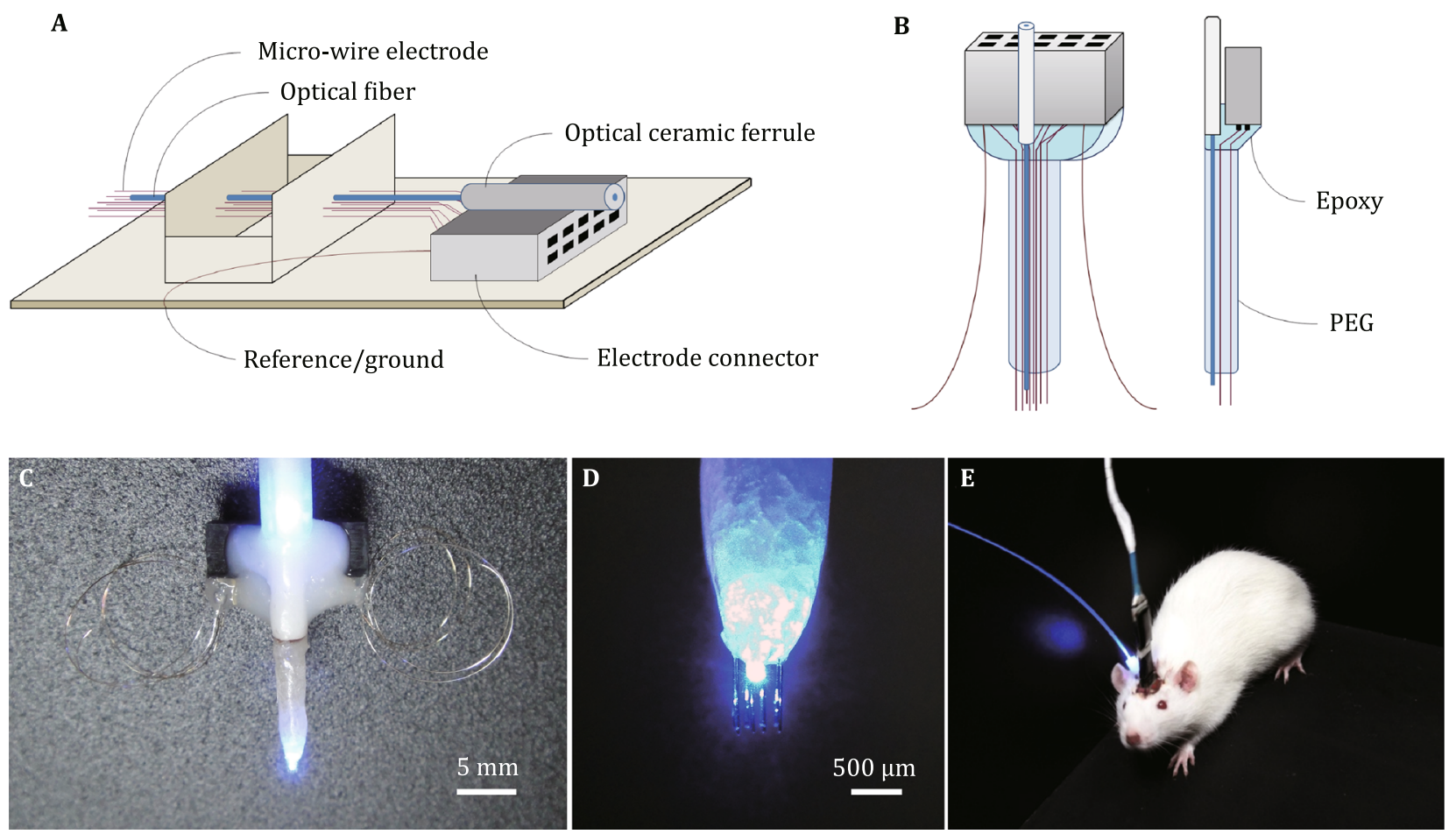

Fig. 1 Fabrication of microwire optrode arrays. A Fabrication process for an eight-channel microwire optrode array using a customdesigned mold. B, C A schematic diagram (B) and photo (C) of an eight-channel microwire optrode array. D Tip of the microwire optrode array (enwrapped by PEG). E Optogenetic stimulation and electrophysiological recording using a microwire optrode array implanted in a free-moving rat

microwires. Each tetrode was threaded through a silica tube (inner diameter $100 \mu \mathrm{m}$, outer diameter $164 \mu \mathrm{m}$, Polymicro, USA). In an optrode bundle, eight tetrodes were arranged around an optical fiber, and the centerto-center spacing between each tetrode and the optical fiber was approximately $200 \mu \mathrm{m}$ (Fig. 2C). Then, the optrode bundles were glued onto the movable screw nut on the microdrive scaffolds. Additional optrode bundles can be integrated with the microdrive system to construct a multisite drivable optrode array (Fig. 2D-F).

\section{Electrodeposition}

\section{Electrodeposition of Pt}

All electrochemical experiments were performed using a potentiostat (Gamry Reference 600, USA). The electrodes were mounted in a three-electrode cell with a saturated calomel electrode (SCE) as the reference electrode and a large-area platinum electrode as the counter electrode. The electrodeposition solution was prepared by dissolving $\mathrm{PtCl}_{4}$ in $100 \mathrm{mmol} / \mathrm{L} \mathrm{HCl}$ solution to a concentration of $5 \mathrm{mmol} / \mathrm{L}$. The prepared solution can be used for at least six months after preservation at $4{ }^{\circ} \mathrm{C}$. Platinum nanoparticles were deposited on the recording sites of the optrode in the deposition solution via a simplified cathodic reaction at a potential of $-0.25 \mathrm{~V}$ (vs. SCE) applied by a Gamry potentiostat. The electrodeposition process can be described by the following equation:

$\mathrm{Pt}^{4+}+4 \mathrm{e}^{-}=\mathrm{Pt}$.

\section{Electrodeposition of iridium oxide $\left(\mathrm{IrO}_{2}\right)$}

The electrodeposition solution was prepared as described in previous studies (Lu et al. 2009b; Zhong et al. 2014). Briefly, oxalic acid $\left(\mathrm{H}_{2} \mathrm{C}_{2} \mathrm{O}_{4}\right)$ was slowly dissolved to a concentration of $100 \mathrm{mmol} / \mathrm{L}$ in a $3 \mathrm{mmol} / \mathrm{L}$ hydrogen hexachloroiridate $\left(\mathrm{H}_{2} \mathrm{IrCl}_{6}\right)$ aqueous solution, and $\mathrm{H}_{2} \mathrm{O}_{2}$ $(2 \% \mathrm{v} / \mathrm{v})$ was added to form a stable complex of iridium oxide. Then, the $\mathrm{pH}$ of the solution was adjusted to 10.5 by gradually adding $\mathrm{K}_{2} \mathrm{CO}_{3}$. The resulting solution was allowed to age for one week at room temperature until it turned dark blue. The deposition solution can be used for more than two months after preservation at $4{ }^{\circ} \mathrm{C}$. Thin $\mathrm{IrO}_{2}$ films were formed on the recording sites in the stabilized electrodepositing solution by cyclic voltammetry scanning between 0.05 and $0.55 \mathrm{~V}$ (vs. SCE) at $100 \mathrm{mV} / \mathrm{s}$ with a Gamry potentiostat 

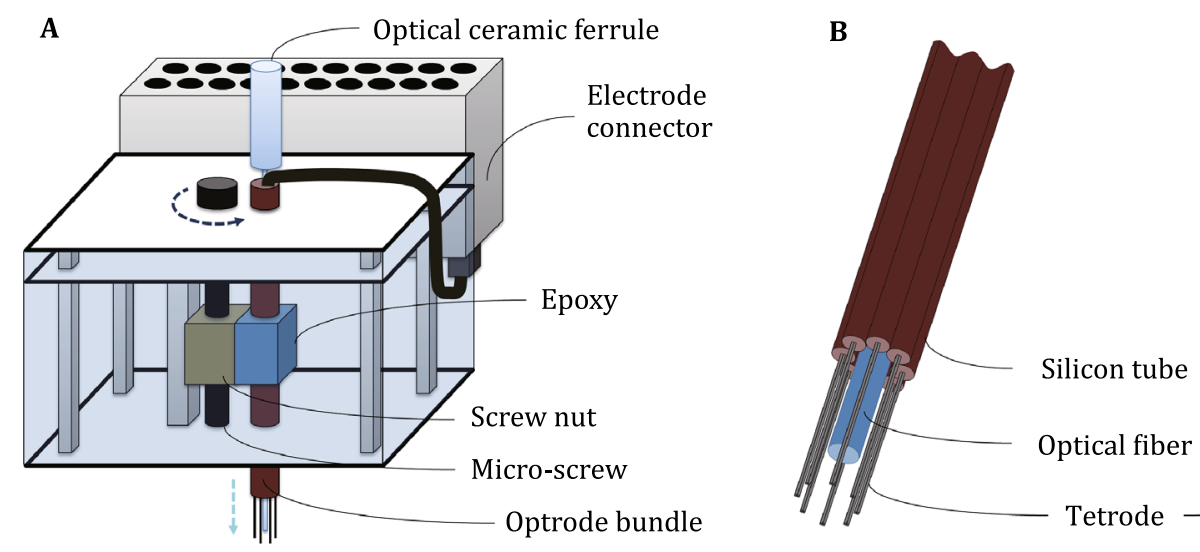

C

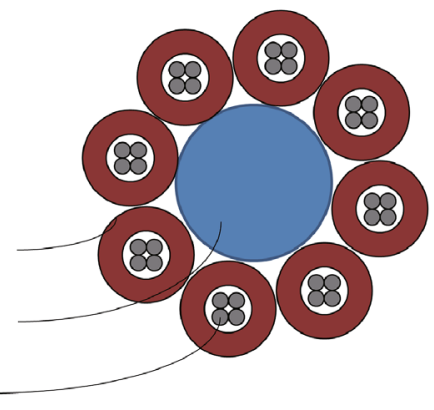

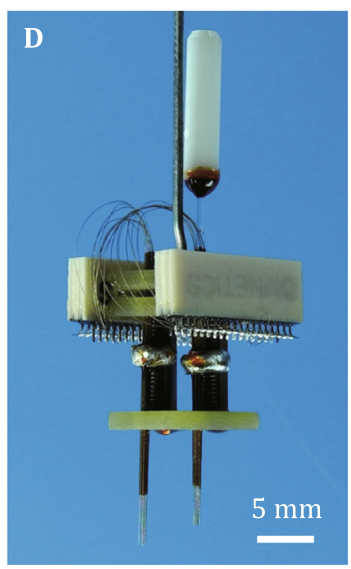
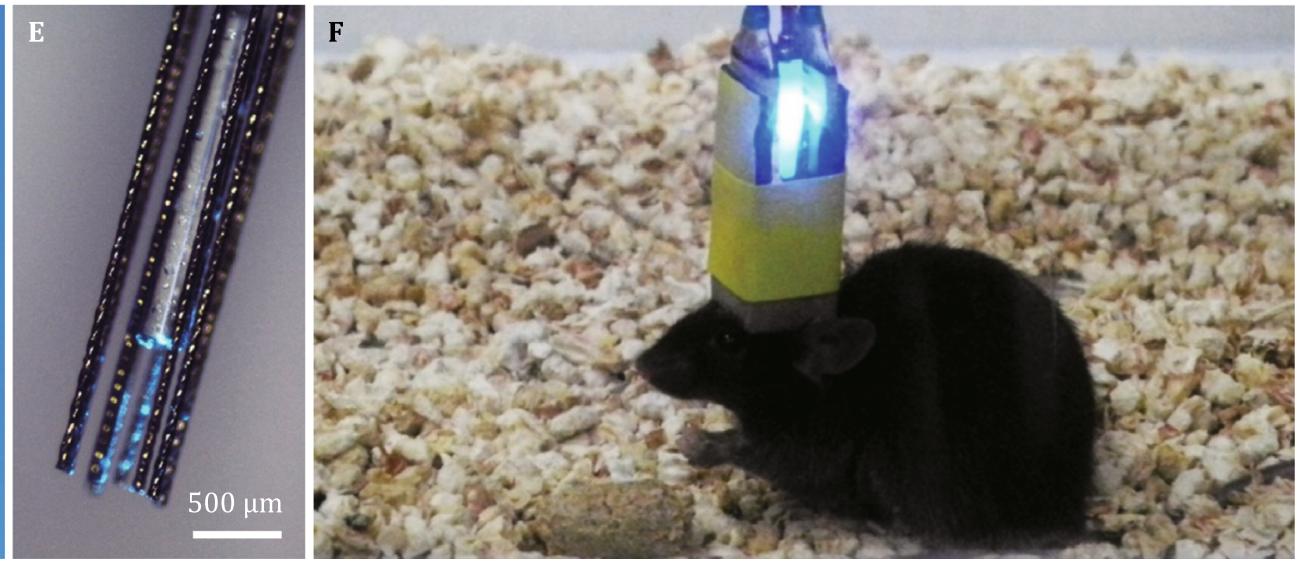

Fig. 2 Design of multisite drivable optrode arrays. A Schematic diagram of a 32-channel drivable optrode array. B, C Detailed schematic diagram (B) and top view (C) of the optrode tip. D, E Photos of a 64-channel multisite drivable optrode array (D) and the optrode tip (E). F Optogenetic stimulation and electrophysiological recording using a multisite drivable optrode array implanted in a freely moving mouse

(Fig. 3). The electrodeposition process can be described by the following equation (Lu et al. 2009b):

$$
\left[\mathrm{Ir}(\mathrm{OH})_{4} \mathrm{C}_{2} \mathrm{O}_{4}\right]^{2-}-2 \mathrm{e}^{-}=\mathrm{IrO}_{2}+2 \mathrm{CO}_{2}+2 \mathrm{H}_{2} \mathrm{O} \text {. }
$$

\section{Electrodeposition of composite conducting polymers (CPS)}

Poly(vinyl alcohol)/poly(acrylic acid) interpenetrating polymer networks (PVA/PAA IPNs) were synthesized following the approach described in previous studies (Lu et al. 2012; Zhong et al. 2017). Briefly, an aqueous PVA solution (2 wt\%, $M_{\mathrm{W}} 89,000-98,000 \mathrm{~g} / \mathrm{mol}$, SigmaAldrich, USA) was placed in a round-bottom flask, and acrylic acid (AA) monomer was added under magnetic stirring to a ratio of $60 \mathrm{~mol} \%$ (moles of AA monomer per mole of PVA repeat unit). Ammonium persulfate (Degussa-AJ, China) was added to the flask to a concentration of $1000 \mathrm{ppm}$ as an initiator. Then, the mixed solution was purged with $\mathrm{Ar}$ to remove $\mathrm{O}_{2}$, and the flask was sealed and immersed in an oil bath at $80{ }^{\circ} \mathrm{C}$. The reaction was allowed to take place for $72 \mathrm{~h}$, and the resulting polymer solution was filtered to remove undissolved solids and bubbles prior to use. The samples were dipped into the homogeneous polymer solution and dried at $60{ }^{\circ} \mathrm{C}$ for $2 \mathrm{~h}$ to form a thin hydrogel film on the surface. The aqueous electrodeposition solution was prepared by dissolving $10 \mathrm{mmol} / \mathrm{L}$

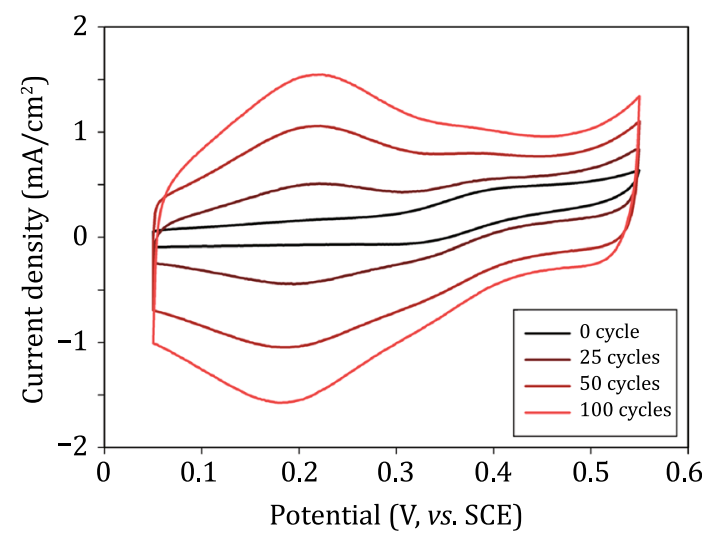

Fig. 3 The deposition of $\mathrm{IrO}_{2}$ under repetitive potential cycling between 0.05 and $0.55 \mathrm{~V}$ (vs. SCE) in an iridium complex $\left(\left[\operatorname{Ir}(\mathrm{OH})_{4} \mathrm{C}_{2} \mathrm{O}_{4}\right]^{2-}\right)$ solution 
3,4-ethylenedioxythiophene monomer (EDOT, SigmaAldrich, USA), $250 \mathrm{mmol} / \mathrm{L}$ poly(sodium 4-styrenesulfonate) (PSSNa, $M_{\mathrm{W}} 70,000 \mathrm{~g} / \mathrm{mol}$, Sigma-Aldrich, USA), $10 \mu \mathrm{g} / \mathrm{mL}$ nerve growth factor (NGF, Sigma-Aldrich, USA), and $10 \mathrm{mmol} / \mathrm{L}$ dexamethasone sodium phosphate (DEX, NIFDC, China). The coated substrates were then immersed in the electrodeposition solution for at least $1 \mathrm{~h}$ prior to use. PEDOT/PSS/NGF/DEX films were electrodeposited onto the hydrogel-coated substrates using a Gamry potentiostat at $0.9 \mathrm{~V}$ (vs. SCE), forming the composite CP films.

\section{Physicochemical characterizations}

Cyclic voltammetry (CV) measurements were performed within the safe potential window in a testing solution at a scan rate of $50 \mathrm{mV} / \mathrm{s}$ using a Gamry potentiostat. Electrochemical impedance spectra (EIS) of the recording sites were measured at their open circuit potentials in artificial cerebrospinal fluid (ACSF) using a Gamry potentiostat with a $25-\mathrm{mV}$ (rms) AC sinusoid signal in the frequency range $100 \mathrm{kHz}$ to $1 \mathrm{~Hz}$. The morphologies of the electrodeposited surfaces were examined using an extremely high-resolution scanning electron microscope (XHRSEM, FEI Magellan 400L, USA) operated at $5 \mathrm{kV}$. In order to facilitate the observation of the modified surfaces, the recording sites of the optrodes were coated with epoxy. The samples were sputtered with a layer of iridium prior to use.

\section{Characterization of Pt-deposited electrodes}

After Pt electrodeposition, the redox currents in the $\mathrm{CV}$ curves were significantly increased (Fig. 4A), suggesting an enlarged double-layer capacitance. The electrochemical impedance at $1 \mathrm{kHz}$ is a critical characteristic parameter for neural electrodes, as this frequency is relevant to the electrical activity of neurons. The average impedance value at $1 \mathrm{kHz}$ was decreased from $1.52 \mathrm{M} \Omega$ to $11.96 \mathrm{k} \Omega, \sim 99 \%$ lower than the unmodified electrode (Fig. 4B), possibly due to the increase in pseudo-capacitance after modification. The surface of the Pt-deposited layer contains numerous nanoparticles that aggregated into sub-micrometer structures. This rough surface significantly increased the effective surface area at the electrode/electrolyte interface and, as a consequence, decreased the electrochemical impedance of the electrode (Fig. 4C).

\section{Characterization of $\mathrm{IrO}_{2}$-deposited electrodes}

No significant current peak was observed in the background CV curve of the undeposited electrode. After cyclic voltammetric scanning in the electrodeposition solution for 100 cycles, two pairs of peaks correlating to redox reactions of iridium oxides appeared at 100 and $500 \mathrm{mV}$, respectively (Fig. 5A). Besides, the electrode impedance at $1 \mathrm{kHz}$ decreased from $2.71 \mathrm{M} \Omega$ to $148 \mathrm{k} \Omega, \sim 95 \%$ lower than that of the unmodified electrode (Fig. 5B). The surface of the electrode substrate presented a homogeneous and flat morphology (Fig. 5C, D). By comparison, the deposited $\mathrm{IrO}_{2}$ film was composed of numerous $20-50 \mathrm{~nm}$ particles, and the film exhibited a more porous structure and increased roughness (Fig. 5E, F), which is beneficial for the improvement of the electrochemical performance.

\section{Characterization of composite CP-deposited electrodes}

The CV measurement of the composite CP-modified electrode shows the emergence of an anodic peak and a cathodic peak at -300 and $-400 \mathrm{mV}$ (vs. SCE), respectively (Fig. $5 \mathrm{~A})$. In the high-frequency range $\left(10^{2}-\right.$ $10^{5} \mathrm{~Hz}$ ), the impedance of the composite CP-modified electrode was almost independent of frequency. The average impedance value at $1 \mathrm{kHz}$ was $\sim 95 \%$ lower than the impedance of the unmodified electrode (Fig. 5B). The drastic increase in charge-storage capacity and reduction in impedance after modification is possibly due to the increase of the pseudo-capacitance and effective surface area (Fig. 5C) at the electrode/electrolyte interface.

\section{Optogenetic study}

\section{Seizure detection and inhibition}

VGAT-ChR2(H134R)-EYFP BAC transgenic mice were anesthetized with urethane $(1.5 \mathrm{~g} / \mathrm{kg})$ and fixed in a standard stereotaxic frame. Holes were drilled through the skull, and the multisite optrodes were implanted. Optrode bundles were directed toward the dentate gyrus/hilus (DGH, stereotaxis atlas coordinates: AP $-2.06 \mathrm{~mm}, \mathrm{ML}+1.35 \mathrm{~mm}$, DV $+2.10 \mathrm{~mm}$ ) and primary motor cortex (M1, AP $+1.80 \mathrm{~mm}$, ML $+1.80 \mathrm{~mm}$, DV $+1.80 \mathrm{~mm}$ ), respectively.

Kainic acid (KA) was dissolved in PBS at $0.30 \mathrm{mg} / \mathrm{mL}$, and then $550 \mathrm{nl}$ of the KA solution was unilaterally injected into the dorsal hippocampus using a micropump to induce an acute status epilepticus. Electrophysiological recordings were performed using a 64-channel neural acquisition processor (Plexon, USA). After the onset of seizures, $473 \mathrm{~nm}$ blue light pulses $\left(10 \mathrm{~mW} / \mathrm{mm}^{2}\right.$, $5 \mathrm{~ms}$ pulses at $130 \mathrm{~Hz}, 1 \mathrm{~min}$ on and $5 \mathrm{~min}$ off) were directed into the implanted optrode array for optogenetic stimulation. 
A

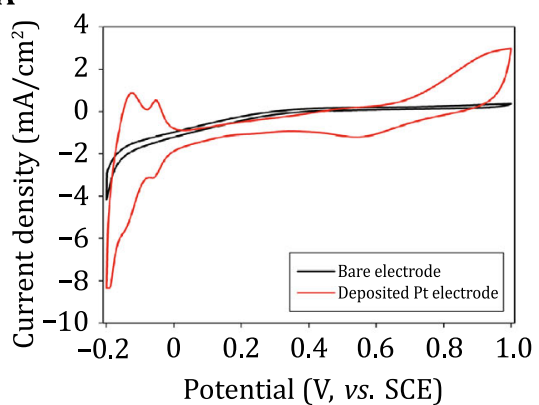

B

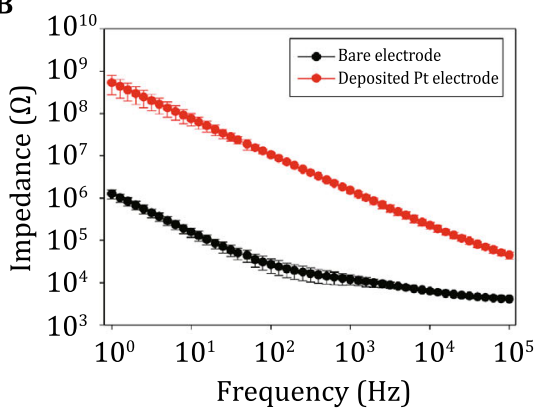

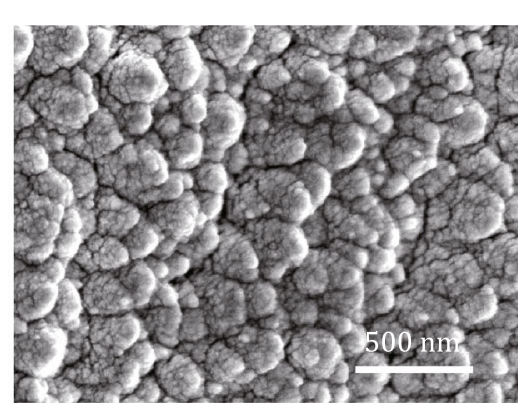

Fig. 4 Characterization of platinum particles. A Cyclic voltammogram of an optrode before (black) and after platinum deposition (red) at a sweep rate of $50 \mathrm{mV} / \mathrm{s}$ in $0.1 \mathrm{~mol} / \mathrm{L} \mathrm{HCl}$. B Bode plot of electrochemical impedance spectra of an optrode before (black, $n=4)$ and after platinum deposition (red, $n=4$ ) in ACSF, data are shown as mean \pm SD. C SEM image of the optrode tip after platinum deposition

A
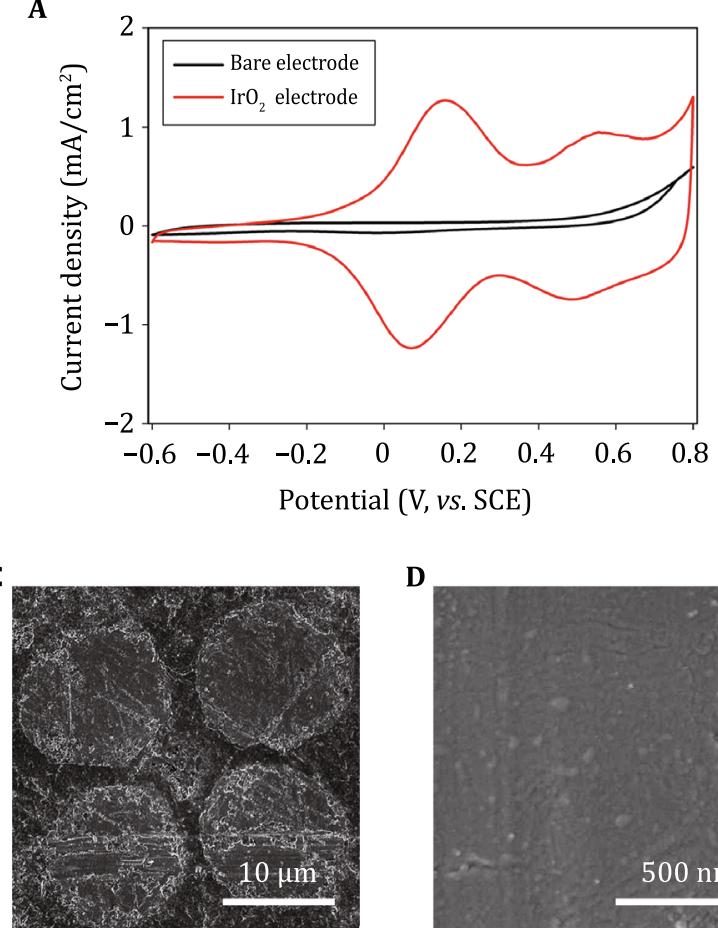

D

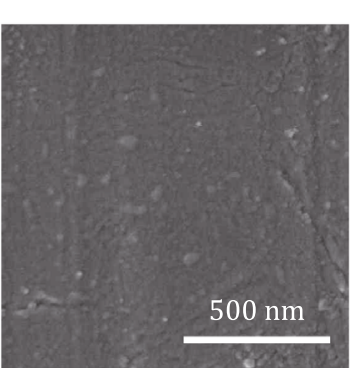

B

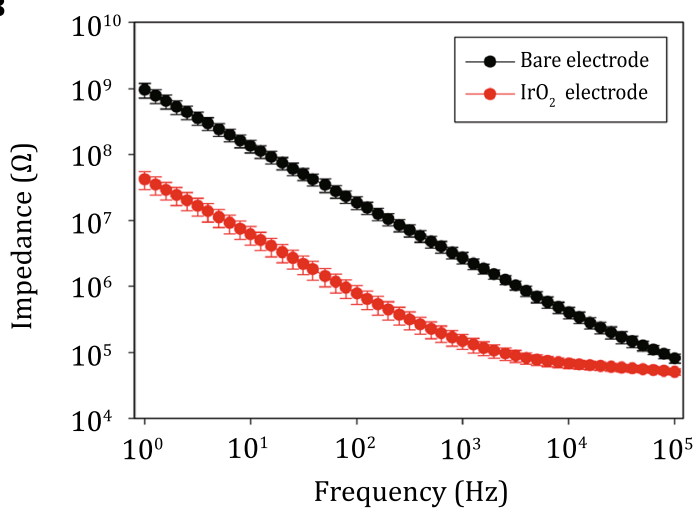

$\mathbf{E}$

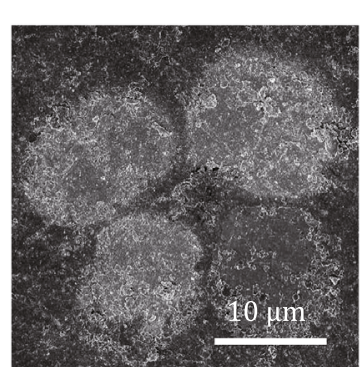

$\mathbf{F}$

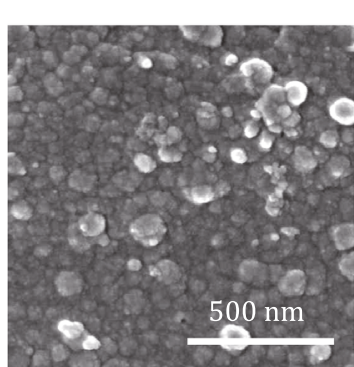

Fig. 5 Characterization of $\mathrm{IrO}_{2}$. A Cyclic voltammogram of an optrode before (black) and after $\mathrm{IrO}_{2}$ deposition (red) at a sweep rate of $50 \mathrm{mV} / \mathrm{s}$ in PBS. B Bode plot of electrochemical impedance spectra of an optrode before (black, $n=4)$ and after $\mathrm{IrO}_{2}$ deposition $($ red, $n=4)$ in ACSF, data are shown as mean \pm SD. C-F SEM images of the optrode tip before (C, D) and after IrO ${ }_{2}$ deposition $(\mathbf{E}, \mathbf{F})$

\section{Data analysis}

Neural electrophysiological data for all recording channels were bandpass filtered. Multi-unit recordings were high-pass filtered $(300 \mathrm{~Hz})$ with a Bessel filter for the detection of spikes. The threshold for spike detection was set to -4.5 SD (standard deviations) and the spike waveforms were measured in a time window $1400 \mu \mathrm{s}$ long and beginning $150 \mu$ s before threshold crossing.
Local field potentials (LFPs) were sampled at $1 \mathrm{kHz}$ and bandpass filtered at 1-300 Hz. Data analyses were performed with a custom software written in MATLAB (MathWorks, USA) and NeuroExplorer (Nex Technologies, USA), and the mean-squared values of the multiunit activities, $60 \mathrm{~s}$ before and $300 \mathrm{~s}$ after optogenetic stimulation (10 s bins), were calculated. LFPs were filtered in beta $(12-30 \mathrm{~Hz})$ and gamma bands $(30-80 \mathrm{~Hz})$. The squared values of the filtered signals, $60 \mathrm{~s}$ before 
and $300 \mathrm{~s}$ after stimulation (10 s bins), were calculated for each band. The power measured before stimulation was averaged ( 6 bins, $60 \mathrm{~s}$ ). The power in each bin measured after stimulation was compared with the average power before stimulation by a one-side paired $t$ test to investigate whether optical stimulation reduced LFP power.

\section{Histological study}

\section{Sample preparation and implantation procedures}

After being anesthetized with $1 \%$ phenobarbital sodium solution $(1 \mathrm{ml} / 100 \mathrm{~g})$, the rats were immobilized in a stereotaxic frame for sample implantation. A midsagittal incision was made in the scalp, and two holes were carefully bored in each animal at locations $-4.0 \mathrm{~mm}$ anterior and $\pm 3.0 \mathrm{~mm}$ lateral to the bregma. The brains were slowly implanted with the samples: Pt/Ir dummy probes (diameter $100 \mu \mathrm{m}$, uninsulated) as the control group and the composite CP-deposited Pt/Ir dummy probes as the testing group. Finally, the implants were fixed to the skull by gluing the cap to the skull surface with a medical adhesive (Fuaile, China), and the skin was sutured shut with monofilament nylon.

\section{Tissue preparation and histological analysis}

Six weeks after implantation, the rats were sacrificed and immunohistological analyses were performed as described previously (Lu et al. 2009a, 2010; Zhong et al. 2017). Briefly, the rats were perfused transcardially with $0.1 \mathrm{~mol} / \mathrm{L}$ PBS followed by $4 \%$ paraformaldehyde in $0.1 \mathrm{~mol} / \mathrm{L} \mathrm{PBS}$, and the brains were removed and fixed at $4{ }^{\circ} \mathrm{C}$ for two days. Subsequently, the block tissue around the implant was paraffin-embedded, and horizontal sections (30 $\mu \mathrm{m}$ thick) were prepared from all the brain samples. Antibodies against GFAP and NeuN (both from Abcam, USA) were used to label astrocytes and mature neurons, respectively. Fluorescence images were obtained using an Olympus IX71 inverted fluorescence microscope. Quantitative analysis was performed using custom software developed in MATLAB. The staining intensities of GFAP and NeuN were calculated as a function of distance up to the implant surface. The results shown are the average intensity profiles of the analyzed area within a distance of $250 \mu \mathrm{m}$ from the implant/neural-tissue interface. All data are presented as mean \pm standard error of the mean. The differences in staining intensities of various implants at the same distance were analyzed by performing independent sample $t$ tests using SPSS 16.0 (SPSS, USA).

\section{RESULTS AND DISCUSSION}

\section{Fabrication and surface modification of optrode arrays}

In order to integrate optogenetic stimulation with electrophysiological readout methods, chronically implantable optrode arrays were designed and fabricated. An optrode mold was custom made for arranging the microwires and optical fibers (Fig. 1). Aided by this mold, the recording and stimulation channels can be customized to different experimental designs, which greatly simplified the fabrication process. In order to record neural activity from a large number of individual cells in relatively deep brain areas, drivable optrode arrays were designed based on previous work (Lin et al. 2006). The position of optrode bundles on the microdrive scaffold can be conveniently formatted according to different experimental needs (Fig. 2). This lightweight (typically $<2$ g) optrode array can be used to explore the causal, temporally precise, and behaviorally relevant interactions of neurons in multiple brain regions of freely moving mice.

As the electrode (recording site) is a key readout element of an optrode, its performance is crucial for optogenetic investigations. For improved spatial resolution and signal quality during electrophysiological recording, neural electrodes must meet the requirements of small size and low interface impedance (Cogan 2008). However, because the electrochemical impedance at the interface is inversely proportional to the square of the electrode diameter, balancing the impedance and the size of a neural electrode is a difficult task.

One of the most commonly applied strategies involves using high-capacitance electroactive materials to decrease the charge-transfer resistance at the electrode/electrolyte (electrode/neural-tissue) interface. Therefore, we modified the recording sites of optrodes with $\mathrm{Pt}$ nanoparticles, $\mathrm{IrO}_{2}$, and composite $\mathrm{CPs}$, respectively (Figs. 4, 5, 6). After electrodeposition, the redox currents in the cyclic voltammograms of the recording sites were significantly increased, while the electrochemical impedances at $1 \mathrm{kHz}$ were $95 \%-99 \%$ lower than the unmodified sites. This is possibly due to the increase in the pseudo-capacitance after modification, which may be beneficial for decreasing background thermal noise and improving signal quality during electrical recording.

It should be mentioned that the impedance at $100 \mathrm{kHz}\left(Z_{100 \mathrm{kHz}}\right)$ for the Pt-deposited electrode was approximately $90 \%$ lower than that for the undeposited electrode. As the electrode impedance at particularly 
A

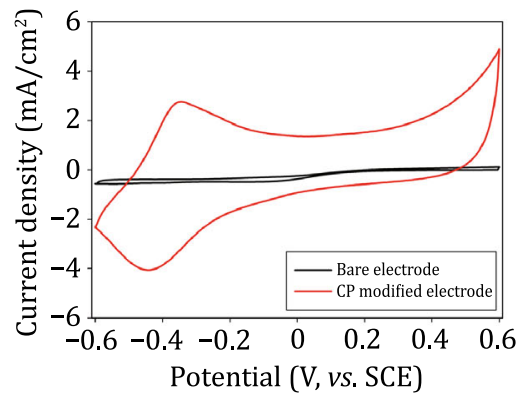

B

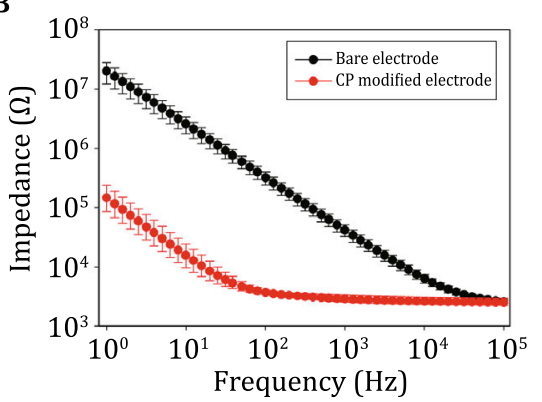

C

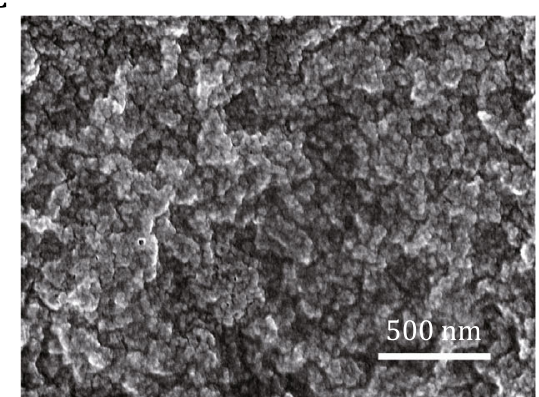

Fig. 6 Characterization of conducting polymer (CP) films. A Cyclic voltammogram of an optrode before (black) and after CP deposition (red) at a sweep rate of $50 \mathrm{mV} / \mathrm{s}$ in PBS. B Bode plot of electrochemical impedance spectra of an optrode before (black, $n=6)$ and after CP deposition (red, $n=6$ ) in ACSF. C SEM image of the optrode tip after CP deposition

high frequencies mainly depends on the geometric area of the conductive site, the reduction of $Z_{100 \mathrm{kHz}}$ is probably due to the expanded electrode surface covered with Pt particles after electrodeposition. The expansion of the deposited Pt layers may lead to a short circuit of the neighboring recording sites (especially on tetrodes or other high-density electrode arrays). However, the impedance of the $\mathrm{IrO}_{2}$ electrode and the CP electrode at $100 \mathrm{kHz}$ were comparable with that of the undeposited electrode, implying that the $\mathrm{IrO}_{2}$ and $\mathrm{CP}$ films were not over-expanded during electrodeposition. The electrodepositions of the $\mathrm{IrO}_{2}$ and $\mathrm{CP}$ films are much more controllable than $\mathrm{Pt}$ deposition, which is especially suitable for high-density microelectrode arrays.

\section{In vivo seizure detection and inhibition}

We next verified the feasibility of using the fabricated optrode arrays to investigate the circuit-level mechanisms controlling seizure activities under the modulation of specific neuron subtypes at high temporal resolution in vivo. A customized two-site optrode array was implanted simultaneously into the DGH and M1 of the VGAT-ChR2(H134R)-EYFP transgenic mice. Neural activity in the M1 was recorded as an indicator of behavioral seizures. After the onset of ictal seizures, spontaneous multi-unit bursts and large-amplitude spikes and LFPs were frequently detected in the DGH and M1 (Fig. 7), suggesting hypersynchronization of the affected neurons. We found that selectively activating DGH GABAergic interneurons not only significantly decreased the activity of local neurons $(n=27$ from 4 mice, Fig. 7A), but also inhibited multi-unit firings in the M1 ( $n=22$ from 4 mice, Fig. 7E). During ictal seizures, the LFP power in the DGH and M1 all remarkably increased within a broad frequency range. Optical stimulation of the DGH GABAergic interneurons caused a significant decrease in local LFP activity, especially at the beta-gamma band ( $n=4$ mice, Fig. 7B-D), as well as in the M1 ( $n=4$ mice, Fig. 7F-H). The neuronal activity levels reduced to control baselines (relative LFP powers are close to 1) during optogenetic modulation, which indicates that activating the DGH inhibitory neurons is sufficient to rescue ongoing behavioral seizures.

\section{Histological analysis}

After a neural electrode is implanted into the central nervous system, a host response is subsequently elicited, which results in the encapsulation of the implant by a dense astrocyte layer that separates the implant from the targeted neurons and hinders charge transfer at the electrode/neural-tissue interface. Consequently, the electrode impedance is increased substantially, which causes a decline in signal quality. Furthermore, the inflammatory response might also lead to a loss of neurons adjacent to the implant/neural-tissue interface, which would further deteriorate the performance of the neural electrodes. Therefore, we used Pt/Ir implants as electrodeposition substrates (the control group); these implants feature large electrode areas and can be used conveniently for investigating the responses of the electrode interface to the host tissue. Previous studies demonstrated that the iridium oxide modified implants exhibited a more significant reactive response compared to platinum substrates in vivo (Ereifej et al. 2013). Therefore, to improve biocompatibility at the implant/ neural-tissue interface, we modified the implants using composite CP films, and then tested each of the deposited and control implants. We performed immunochemical analysis on tissue sections of rat brain at six weeks after implantation and evaluated the chronic performance of the composite CP films.

The inflammatory response at the implant/neuraltissue interface was characterized by the expression of 

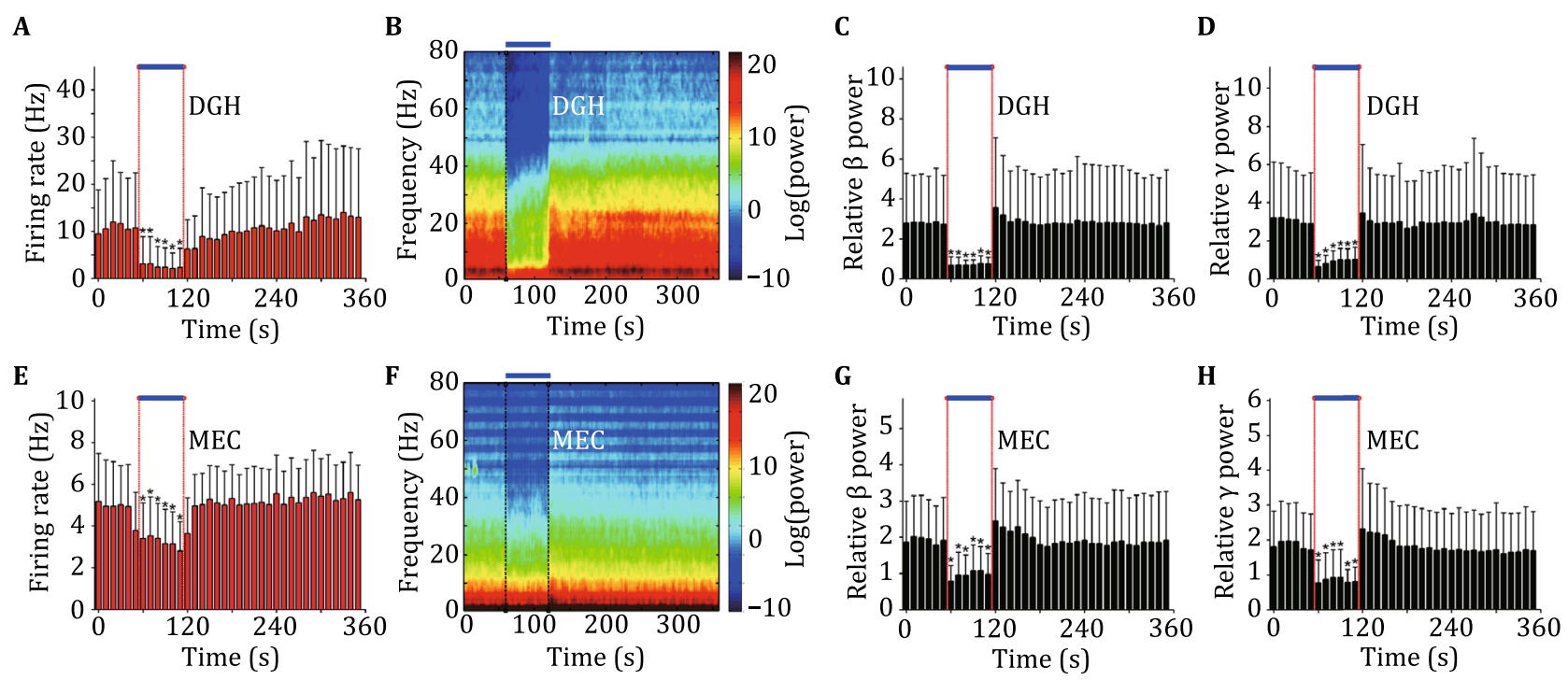

Fig. 7 Optogenetic inhibition of seizures in the intrahippocampal kainate-injected mice using a custom-made multisite optrode array. A, E Averaged multi-unit firing rates recorded in the DG (A, $n=27$ from 4 mice) and M1 (E, $n=22$ from 4 mice). B, F Representative examples of the spectrograms of LFPs in the DG (B) and M1 (F). C, D, G, H Power quantification of DG (C, D, $n=4$ mice) and M1 (G, H, $n=4$ mice) LFPs, the averaged powers were shown in the beta $(\mathbf{C}, \mathbf{G})$ and gamma bands $(\mathbf{D}, \mathbf{H})$, and the values were normalized to the total power in the pre-KA period. Light pulses ( $473 \mathrm{~nm}, 5-\mathrm{ms}$ pulse duration at $130 \mathrm{~Hz}$ ) were delivered into the DG/hilus at time 0 . The thick blue line denotes the 60 -s stimulation period. All data are shown as mean $\pm \operatorname{SEM}\left({ }^{*} p<0.05, t\right.$ test $)$

GFAP, an astrocyte-specific marker. We found that reactivated astrocytes accumulated in and occupied the zone around the implantation site in the control group (Fig. 8A). By comparison, GFAP expression was considerably weaker around the implantation site in the composite CP group (Fig. 8B). Figure 8C shows the quantified GFAP immunohistological intensity profiles of the control and composite CP-modified implants as a function of distance from the interface $(n=7$ in each group). The average thickness of the astroglial encapsulation in the control group was $\sim 150 \mu \mathrm{m}$, whereas it was only $\sim 40 \mu \mathrm{m}$ in the CP-modified group. Statistical results show that the average intensity of GFAP immunostaining in the CP-modified group was significantly lower $(p<0.05)$ than that in the control group up to a distance of $\sim 200 \mu \mathrm{m}$ from the implant interface. Besides, neuronal loss was observed around the implantation site, and this loss was particularly severe in the control group (Fig. 8D). Interestingly, no notable loss of neurons was detected in the composite CP group (Fig. 8E). The quantified NeuN intensity profiles (Fig. 5F, $n=7$ in each group) reveal a severe loss of neurons within an average distance of $\sim 60 \mu \mathrm{m}$ from the implantation site in the control group. However, in the CP-modified groups, the average distance was decreased to $\sim 15 \mu \mathrm{m}$. Statistical analysis suggests that the average intensity of NeuN immunostaining in the modified group was markedly higher than that in the control group $(p<0.05$ within $\sim 60 \mu \mathrm{m})$. Given the inherent merits of the deposited CP film, our data suggest that modification of the electrode with the composite CP film can drastically alleviate the inflammatory response (reduced astroglial intensity) and promote neuronal viability (increased NeuN intensity) around the implant site. This implies that the composite CP film can improve the implant/neural-tissue interface and is suitable for long-term implantation.

\section{CONCLUSION}

In this study, we have demonstrated the feasibility and advantages of using customized optrode arrays for in vivo optogenetic applications. We designed and fabricated a microwire optrode array and a drivable optrode array, which are suitable for targeting single sites in relatively shallow brain structures and multiple sites in relatively deep structures, respectively. Besides, electrochemical deposition strategies for improving the performance of optrode arrays were also presented, and the physicochemical characteristics of the deposited surfaces were investigated. Platinum particles were deposited by a simplified cathodic electrochemical process, which rapidly decreased the electrode impedance (reduced by $99 \%$ at $1 \mathrm{kHz}$ ) by increasing the double-layer capacitance at the electrode interface. Low impedance and stable $\mathrm{IrO}_{2}$ films were formed on the recording sites under a slow-sweep-rate cyclic 
A

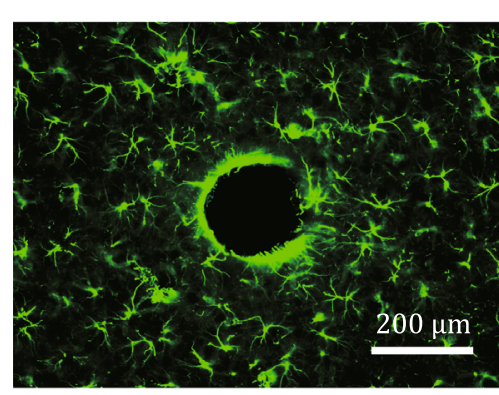

D

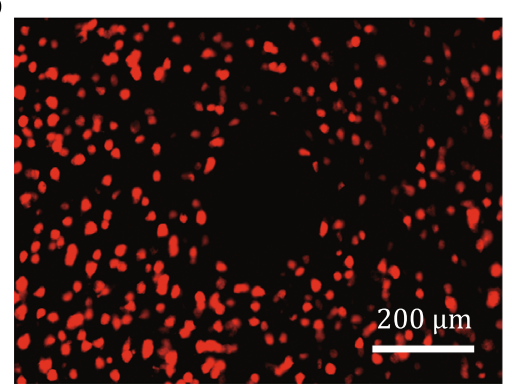

B

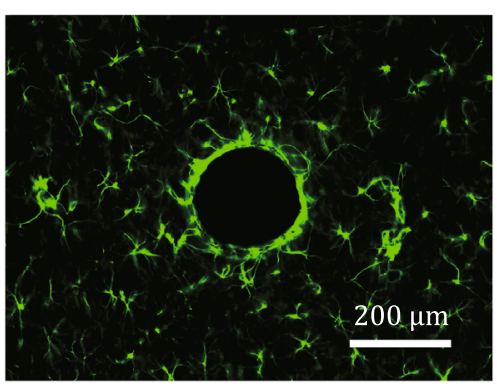

E

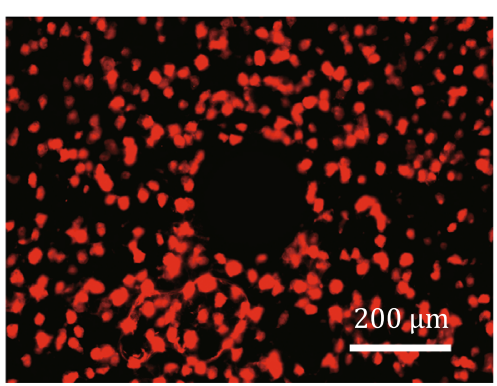

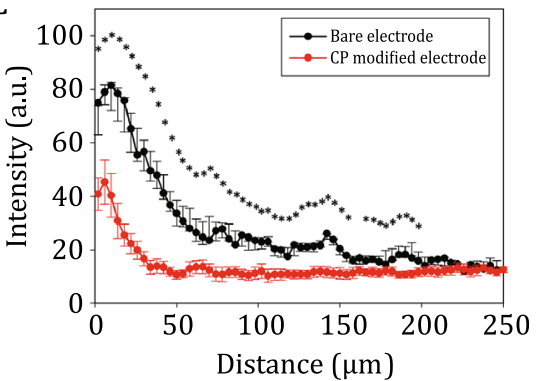

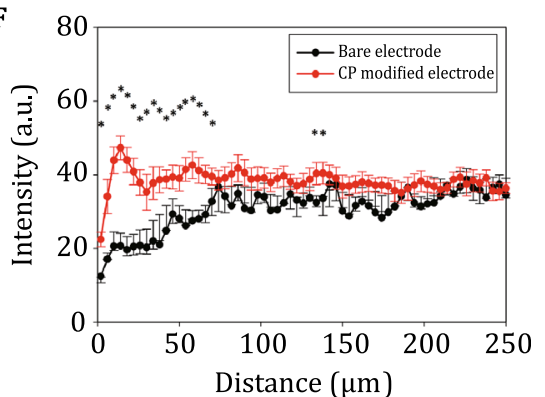

Fig. 8 Histological studies of the composite conducting polymer (CP) films' modified surfaces. Inflammatory response (A-C, GFAP) and neuronal survival (D-F, NeuN) around implants at six weeks post implantation in the rat brain before (A, D) and after composite CP deposition (B, E). Quantitative comparisons of the immunoreactivity between control $(n=7)$ and CP-modified implants $(n=7)$ were performed by using intensity profiles as a function of distance from the implant interface, shown as mean $\pm \mathrm{SEM}(* p<0.05, t$ test)

voltammetric scanning process. The electrodeposition of $\mathrm{IrO}_{2}$ films is highly controllable, which is suitable for high-density optrode arrays. Furthermore, our data suggest that the electrodeposited composite CP films can significantly improve both the electrochemical performance and the biocompatibility of implants. With the aid of our customized optrode arrays, we successfully analyzed the neuronal activity during seizures and inhibited ictal propagation in multiple brain regions in vivo. All of these characteristics demonstrated and discussed here are crucial for the chronic optrode arrays used for precisely timed analyses of neuron subpopulations in freely moving animals. Most importantly, our results provide simple and reliable strategies for the fabrication and surface modification of optrode arrays. Combinations of these aforementioned strategies could help to construct high-performance optrode arrays optimized to the requirements of different experimental conditions. Other new fabrication strategies and novel interface materials, as well as future applications of the optrode arrays, are avenues for further investigations.

Acknowledgements This work was partially sponsored by the NSFC Program (81425010, 31630031, 31700921, 81471164, 31471109), the "Strategic Priority Research Program (XDB0205 0003)", the "Youth Innovation Promotion Association" and the Key Research Program of Frontier Sciences (QYZDB-SSW-SMC056) of the CAS, the Shenzhen Governmental Research Grants (JSGG20160429184327274, JSGG20160428140402911, JCYJ201
60428164440255), the "Guangdong Key Lab of Brain Connectome", the "Shenzhen Engineering Lab of Brain Activity Mapping Technologies", and the "Shenzhen Discipline Construction Project for Neurobiology".

\section{Compliance with Ethical Standards}

Conflict of interest Lulu Wang, Kang Huang, Cheng Zhong, Liping Wang, and Yi Lu declare that they have no conflict of interest.

Human and animal rights and informed consent All institutional and national guidelines for the care and use of laboratory animals were followed.

Open Access This article is distributed under the terms of the Creative Commons Attribution 4.0 International License (http:// creativecommons.org/licenses/by/4.0/), which permits unrestricted use, distribution, and reproduction in any medium, provided you give appropriate credit to the original author(s) and the source, provide a link to the Creative Commons license, and indicate if changes were made.

\section{References}

Abidian MR, Martin DC (2008) Experimental and theoretical characterization of implantable neural microelectrodes modified with conducting polymer nanotubes. Biomater 29:1273-1283

Anikeeva P, Andalman AS, Witten I, Warden M, Goshen I, Grosenick L, Gunaydin LA, Frank LM, Deisseroth K (2012) Optetrode: a multichannel readout for optogenetic control in freely moving mice. Nat Neurosci 15:163-170 
Brown MTC, Tan KR, O'Connor EC, Nikonenko I, Muller D, Luescher C (2012) Ventral tegmental area GABA projections pause accumbal cholinergic interneurons to enhance associative learning. Nature 492:452-456

Chen S, Pei W, Gui Q, Chen Y, Zhao S, Wang H, Chen H (2013) A fiber-based implantable multi-optrode array with contiguous optical and electrical sites. J Neural Eng 10:046020

Cogan SR (2008) Neural stimulation and recording electrodes. Annu Rev Biomed Eng 10:275-309

Dehkhoda F, Soltan A, Ponon N, Jackson A, O'Neill A, Degenaar P (2018) Self-sensing of temperature rises on light emitting diode based optrodes. J Neural Eng 15:026012

Ereifej ES, Khan S, Newaz G, Zhang JS, Auner GW, VandeVord PJ (2013) Comparative assessment of iridium oxide and platinum alloy wires using an in vitro glial scar assay. Biomed Microdevices 15:917-924

George PM, Lyckman AW, LaVan DA, Hegde A, Leung Y, Avasare R, Testa C, Alexander PM, Langer R, Sur M (2005) Fabrication and biocompatibility of polypyrrole implants suitable for neural prosthetics. Biomater 26:3511-3519

Gradinaru V, Mogri M, Thompson KR, Henderson JM, Deisseroth K (2009) Optical Deconstruction of parkinsonian neural circuitry. Science 324:354-359

Grill WM, Norman SE, Bellamkonda RV (2009) Implanted neural interfaces: biochallenges and engineered solutions. Annu Rev Biomed Eng 11:1-24

Iseri E, Kuzum D (2017) Implantable optoelectronic probes for in vivo optogenetics. J Neural Eng 14:031001

Kotov NA, Winter JO, Clements IP, Jan E, Timko BP, Campidelli S, Pathak S, Mazzatenta A, Lieber CM, Prato M, Bellamkonda RV, Silva GA, Kam NWS, Patolsky F, Ballerini L (2009) Nanomaterials for neural interfaces. Adv Mater 21:3970-4004

Kravitz AV, Freeze BS, Parker PRL, Kay K, Thwin MT, Deisseroth K, Kreitzer AC (2010) Regulation of parkinsonian motor behaviours by optogenetic control of basal ganglia circuitry. Nature 466:U622-U627

Kwon KY, Lee H-M, Ghovanloo M, Weber A, Li W (2015) Design, fabrication, and packaging of an integrated, wirelesslypowered optrode array for optogenetics application. Front Syst Neurosci 9:69-69

Li N, Chen T-W, Guo ZV, Gerfen CR, Svoboda K (2015) A motor cortex circuit for motor planning and movement. Nature 519:U51-U88

Lin L, Chen G, Xie K, Zaia KA, Zhang S, Tsien JZ (2006) Large-scale neural ensemble recording in the brains of freely behaving mice. J Neurosci Method 155:28-38

Lu Y, Wang D, Li T, Zhao X, Cao Y, Yang H, Duan YY (2009a) Poly(vinyl alcohol)/poly(acrylic acid) hydrogel coatings for improving electrode-neural tissue interface. Biomater 30:4143-4151

Lu Y, Wang T, Cai Z, Cao Y, Yang H, Duan YY (2009b) Anodically electrodeposited iridium oxide films microelectrodes for neural microstimulation and recording. Sens Actuat B Chem 137:334-339
Lu Y, Li T, Zhao X, Li M, Cao Y, Yang H, Duan YY (2010) Electrodeposited polypyrrole/carbon nanotubes composite films electrodes for neural interfaces. Biomater 31:5169-5181

Lu Y, Li Y, Pan J, Wei P, Liu N, Wu B, Cheng J, Lu C, Wang L (2012) Poly(3,4-ethylenedioxythiophene)/poly(styrenesulfonate)poly(vinyl alcohol)/poly(acrylic acid) interpenetrating polymer networks for improving optrode-neural tissue interface in optogenetics. Biomater 33:378-394

Lu Y, Zhong C, Wang LL, Wei PF, He W, Huang K, Zhang Y, Zhan Y, Feng GP, Wang LP (2016) Optogenetic dissection of ictal propagation in the hippocampal-entorhinal cortex structures. Nat Commun 7:10962

Otis JM, Namboodiri VMK, Matan AM, Voets ES, Mohorn EP, Kosyk O, McHenry JA, Robinson JE, Resendez SL, Rossi MA, Stuber GD (2017) Prefrontal cortex output circuits guide reward seeking through divergent cue encoding. Nature 543:103-107

Schmitt LI, Wimmer RD, Nakajima M, Happ M, Mofakham S, Halassa MM (2017) Thalamic amplification of cortical connectivity sustains attentional control. Nature 545:219-223

Schwartz AB, Cui XT, Weber DJ, Moran DW (2006) Braincontrolled interfaces: movement restoration with neural prosthetics. Neuron 52:205-220

Tovote P, Esposito MS, Botta P, Haudun FC, Fadok JP, Markovic M, Wolff SBE, Ramakrishnan C, Fenno L, Deisseroth K, Herry C, Arber S, Luthi A (2016) Midbrain circuits for defensive behaviour. Nature 534:206-212

Tye KM, Mirzabekov JJ, Warden MR, Ferenczi EA, Tsai H-C, Finkelstein J, Kim S-Y, Adhikari A, Thompson KR, Andalman AS, Gunaydin LA, Witten IB, Deisseroth K (2013) Dopamine neurons modulate neural encoding and expression of depression-related behaviour. Nature 493:537-541

Yizhar 0, Fenno LE, Davidson TJ, Mogri M, Deisseroth K (2011a) Optogenetics in neural systems. Neuron 71:9-34

Yizhar O, Fenno LE, Prigge M, Schneider F, Davidson TJ, O'Shea DJ, Sohal VS, Goshen I, Finkelstein J, Paz JT, Stehfest K, Fudim R, Ramakrishnan C, Huguenard JR, Hegemann P, Deisseroth K (2011b) Neocortical excitation/inhibition balance in information processing and social dysfunction. Nature 477:171-178

Zhang F, Wang LP, Brauner M, Liewald JF, Kay K, Watzke N, Wood PG, Bamberg E, Nagel G, Gottschalk A, Deisseroth K (2007) Multimodal fast optical interrogation of neural circuitry. Nature 446:U633-U634

Zhong C, Zhang Y, He W, Wei P, Lu Y, Zhu Y, Liu L, Wang L (2014) Multi-unit recording with iridium oxide modified stereotrodes in Drosophila melanogaster. J Neurosci Method 222:218-229

Zhong C, Ke D, Wang L, Lu Y, Wang L (2017) Bioactive interpenetrating polymer networks for improving the electrode/neuraltissue interface. Electrochem Commun 79:59-62

Zimmerman CA, Lin Y-C, Leib DE, Guo L, Huey EL, Daly GE, Chen Y, Knight ZA (2016) Thirst neurons anticipate the homeostatic consequences of eating and drinking. Nature 537:680-684 